Original Research

\title{
Pharmacy practice architecture challenges in handling COVID-19 pandemic - sharing experience from a Kosovo pharmacy practice
}

\author{
Arianit Jakupi (iD, Arta Basha Jakupi iD \\ Received (first version): 25-Nov-2021 Accepted: 13-Dec-2021 Published online: 15-Dec-2021
}

\begin{abstract}
Background: Community pharmacies deliver an essential healthcare service, which is broadly established, encompassing the favored and primary contact for community members. The COVID-19 pandemic is continuing to shift the pharmacy roles and services in order to meet the needs of customers. The important value of community pharmacies was more underlined during the pandemic crisis when they were supposed to undertake abundant precautions to avoid cross-infection among users of the space. Their responsibility is also to create a compatible, efficient, and safe environment inside their premises during this outbreak. The built environment is an important share of pandemic solutions and emergency planning preparations. Objectives: The aim of this paper was to explore the preparedness of community pharmacies in Kosovo for the COVID-19 outbreak in regards to pharmacy design modifications while ensuring adequate medicine management, information, and public health. Consequently, at what stage can the pandemic affect the physical and built environment of pharmacies. Methods: The methods for this study include a cross-sectional survey of 104 community pharmacies operating in seven municipalities in Kosovo during the pandemic. Data collection was conducted from October 1 to October 14, 2021, using self-administered online questionnaires. The questionnaire consisted of a combination of closed and open-ended questions, optional statements, and multiple answers. Data were analyzed in SPSS for Windows version 20 using descriptive statistics. Results: The results show that communities should be prepared ahead in regard to the emergency situations and some of these preparations might be also part of the legislation requirements. Although the pandemic exposed many errors in the Kosovo community pharmacy system, it has also discovered a unique possibility for collaboration and novelty. Conclusions: The study concludes with the recommendation for the architectural design of pharmacies to be positioned as an active mediator in the fight against the COVID 19, with a critical lesson aimed at the flexibility of the space to accommodate emergent situations and create alternative areas for use.
\end{abstract}

Keywords

COVID-19; Pharmacy architecture; Community pharmacy; Kosovo

\section{INTRODUCTION}

The coronavirus 2019 (COVID-19) is the most significant public health threat that society has encountered in modern times. On March 12, 2020, the COVID-19 outbreak was declared a pandemic by the World Health Organization. ${ }^{1}$ The new virus presented new challenges and opportunities to healthcare systems worldwide by requiring changes in daily practices to ensure the health and safety of patients and medical staff. ${ }^{2}$

As a vital part of the healthcare system, pharmacies provide medicine, therapeutics, vaccines, and critical health services to the public. ${ }^{3}$ Community pharmacists are the health professionals most accessible to the public and are a cornerstone of primary health care. ${ }^{4}$ Over the last century, significant evolutionary changes have occurred in pharmacy practice in terms of the definition of the pharmacists' role, pharmacy culture, their perceived

Arianit JAKUPI. Prof.Ass. HEI UBT. Faculty of Pharmacy, Department of Drug Management. Kosovo. arianit.jakupi@ ubt-uni.net

Arta Basha JAKUPI*. Prof.Assoc. University of Prishtina, Faculty of Architecture, Department of Architecture. Kosovo.arta.jakupi@uni-pr.edu place within the healthcare system, and their professional role identity. Overall, it is assumed that there is an impact that may influence permanent pharmacist role change following the pandemic. ${ }^{5}$

The research looked at the contributions of frontline pharmacists during the COVID-19 pandemic. Pharmacists undertake the services like dispensing, consultations but as well activities for infection prevent ion in the community pharmacies and highlight how there are differences and limitations because of the different ways the pharmacy is designed. Consequently, the magnitude of pandemics affecting a pharmacy's physical and built environment is still not known.

The principles of infection prevention, control, and social distancing due to COVID 19 are provided in guidelines from different international and national pharmaceutical and healthcare agencies. ${ }^{5,6}$ Guidance for community pharmacies during the COVID-19 response constitutes emergency strategies to support the pharmacy workforce around the world in helping minimize and control the outbreak and in providing advice and care to patients and communities. ${ }^{5,6}$ These guidelines highlight that, pharmacists are required to implement strategies that will be best suited for their pharmacy, based on their risk assessment level. Crucially, because pharmacy is an 
Jakupi A , Jakupi AB. Pharmacy practice architecture challenges in handling COVID-19 pandemic - sharing experience from a Kosovo pharmacy practice. Pharmacy Practice 2021 Oct-Dec;19(4):pagenumber.

https://doi.org/10.18549/PharmPract.2021.4.pagenumber

essential service, it is the responsibility of pharmacists to ensure an optimal supply of medicines, devices, and masks. In addition, pharmacists are actively involved in educating customers about disease prevention, triaging and referring patients whenever required, and medication counseling. Further, and more importantly, services should be consistently provided, thereby ensuring quality without compromising the health and well-being of pharmacy staff. 7,8

Therefore, the preparedness of community pharmacies in Kosovo for the COVID-19 outbreak concerning pharmacybuilt environment design modifications is explored through the conceptual framework model of three layers of pharmacists' roles: medication management, information, and public health. ${ }^{5}$

We hypothesize that COVID-19 has changed these roles, emphasizing pharmacists' contributions to ensuring a safe and well-organized environment. However, the COVID-19 impact on pharmacy design is subject to adequate realization of the emergency guidelines provided by different agencies and their scope of adaption. For some pharmacies, it may have been a catalyst for a new design or just a minor alteration, whereas, for others, it could have been challenging and thought-provoking.

The medication management domain of the pharmacist during COVID-19 revived central pharmacists' roles, with some of them adapting remote service deliveries as a means of responding and continuing to serve the community and meet their medical needs. In contrast, other 'traditional' medication management roles (e.g., drug supply, managing shortages, and compounding) have also been highly emphasized in most cases during the COVID-19 pandemic. ${ }^{5}$

The information domain is central to acknowledging pharmacists as information professionals who share information to provide patient care. Yet this has been expanded, as the pandemic has highlighted pharmacists as essential healthcare providers for a global health crisis and identified them as a trusted information source providing valuable input for guidance, education, and policy. ${ }^{5}$

The Public Health domain shows the emerging pharmacists' roles in public health and protects and promotes safety. COVID-19 pandemic has shifted the perception of pharmacy practice and enhanced the acknowledgment of the essential pharmacists' roles in a public health crisis. ${ }^{5}$

The built environment is critical for pandemic solutions and emergency preparedness. ${ }^{9}$ The community pharmacy facilities and their design may or may not be quickly adaptable, regardless of actual spatial capacity. ${ }^{9}$ Throughout history, the design and inhabitation of physical space has been a primary defense against epidemics. People have redesigned cities, infrastructure, architecture, and interiors, all in the name of minimizing the risk of infectious disease ${ }^{10}$ Various preventive aids have been suggested to limit the spread of infection among societies-wearing facial masks, practicing hand hygiene, and social distancing are among the top recommended measures. ${ }^{11-13}$ There has been much innovative repurposing due to the COVID 19 measures, but many cases are severely constrained by a physical infrastructure that fails to match newly identified needs. ${ }^{14}$

According to the legal and regulatory framework for community pharmacies in the WHO European Region, several countries have defined a minimum area required for pharmaceutical spaces. This varies between countries and often depends on the pharmacy services performed. For instance, in Montenegro, the minimum space for a pharmacy is $50 \mathrm{~m} 2$, while in Germany, the minimum area is $110 \mathrm{~m} 2$, associated with the list of the different sections or rooms required. ${ }^{4}$ In Kosovo, the minimum conditions necessary for pharmacy licensing are a total area of min $42 \mathrm{~m} 2 .^{15}$ This space poses a challenging line for design approaches to include all national and international emergency COVID 19 preventive guidelines.

Moreover, depending on the services provided by the pharmacy, additional premises and equipment might be needed. Among these services other premises may be required (separate area and storage): sterile production of medicines; supply of hospitals with medicines; supply and individual preparation of weekly dosages for nursing homes; automatic blister services; selling medicines online and shipping medicines via the mail service. ${ }^{4}$ Whereas some services, e.g., medicines online and shipping medicines per post, which proved to be very effective during pandemics, are prohibited by law in Kosovo (Kosovo Ministry of Health, 2014). Therefore, using all forms of professional expertise and specialties is vital to a quick resolution of the crisis by respecting the local laws, avoiding unintended consequences in the short term, and embracing flexible design in the healthcare facilities in the longer term. ${ }^{9}$

Architects in general, and specifically health care architects and certified health facility engineers, are vital and valuable resources for rapid adjustments to current health care and non-healthcare infrastructurerepurposing old health care buildings, erecting temporary structures, and considering emergency preparedness in our building's codes and design guidelines. ${ }^{9}$ In addition, these experts can fast assess existing space utilization in community pharmacies to maximize existing areas for use.

Multiple search engines did not reveal any studies about Kosovo's community pharmacy design challenges during the COVID-19 pandemic. However, there has been a single study about community pharmacy services during COVID-19 in Kosovo. ${ }^{16}$ Furthermore, some of the findings from this study have been taken further and used to build 
Jakupi A , Jakupi AB. Pharmacy practice architecture challenges in handling COVID-19 pandemic - sharing experience from a Kosovo pharmacy practice. Pharmacy Practice 2021 Oct-Dec;19(4):pagenumber.

https://doi.org/10.18549/PharmPract.2021.4.pagenumber

an argument for the current study.

Kosovo was one of the last European countries to report their first cases of COVID-19. The first two cases were reported on March 13, both of whom had traveled from Italy. In Kosovo, a country of 1.8 mil residents, ${ }^{17}$ from January 3, 2020, to September 20, 2021, there have been 159,064 confirmed cases of COVID-19 with 2,889 deaths, reported to WHO. As of September 18, 2021, a total of $1,147,803$ vaccine doses have been administered. ${ }^{18}$ Since then, the government has taken strict measures to limit the spread of the disease; these include: curfew during certain times (ranging from partial to total lockdown) and prohibition of mass gatherings, free treatment, and quarantine facilities even for violators of residence, field screening, and contact tracing, and spreading of the culture of hygiene and social distancing among citizens and residents, especially when visiting crowded areas. ${ }^{19}$ However, given that there is still a large number of nonvaccinated people and there is no current $100 \%$ effective treatment available, we must turn to non-pharmacologic means-including social distancing, isolation, and quarantine-which depend on the built environment. ${ }^{9}$

The objective of this study was to investigate the preparedness of community pharmacies in Kosovo for COVID-19 in regards to pharmacy design modifications, providing a safe environment to avoid the transmission of infection. Furthermore, the study shares the experience of the Kosovo community pharmacies regarding the outbreak supplied by these pharmacies and the convenience and quality of preventive measurement against the infection adopted by them.

\section{METHODS}

The study protocol is approved by the Chamber of Pharmacists in Kosovo (KPC). Community pharmacies in Kosovo were assessed for their preparedness for the COVID-19 pandemic using a cross-sectional online survey from October 1 to October 14, 2021.The study confidentiality statement was explained to participants at the beginning of the survey.

The development of the questionnaire was based on existing literature (specifically that published by the WHO, CDC, Kosovo Pharmacists' Chamber, National Institute of Public Health, the Ministry of Health and other Kosovo government public health guidelines and from the International Pharmaceutical Federation (FIP). The questionnaire included 52 questions divided into four sections. The first section comprised six questions covering pharmacists' demographics, such as position, years of experience, gender, pharmacy ownership, and pharmacy practice location. The second section contained 16 closed questions (yes/no answer) and multiple-choice questions covering the medical management domain related to the COVID-19 pandemic in the pharmacy practice: (1) area destination, (2) services, (3) different measurements. The third section is comprised of information domain with six open-ended questions and multiple-choice questions: (1) practice surrounding patient education, (2) knowledge and awareness. The fourth section contained the public health sphere, with 24 questions open-ended questions and multiple-choice questions: (1) available space, (2) facilitators, (3) barriers, (4) space modifications. Finally, the last three sections comprised of closed-ended questions predominantly, although some questions allowed respondents to add any further comments on the topic at the end of the questionnaire.

A pilot of 60 questions was performed through the questionnaire development. As a result of feedback obtained during the pilot, several changes were made to the questionnaire, namely the addition and removal of specific questions and modifications to the formatting-the final questionnaire comprised 52 questions in four sections. The first section contains demographics questions, and the last three sections covering the domains under investigation. Data were collected using a self-administered questionnaire. Invitations to participate in the research were distributed electronically through the KPC account to pharmacists in Kosovo. The electronic submission keeps participant data anonymized. As such, the survey design had the advantages of capturing responses from individual personnel covering an extensive geographical range, with great rapidity. The present article covers three domains covered in the survey, including the general demographic data, comprising 52 questions. These three domains are (1) medical management, (2) information, (3) public health. The results have been divided into three domains as well, as relevant to the relative directions of the study's objectives.

Data were analyzed in SPSS for Windows version 20 using descriptive statistics.

\section{RESULTS}

Pharmacists completed one hundred and four questionnaires within two weeks, comprising the study sample. Respondents covered all seven municipalities of Kosovo, with the most significant respondents coming from the capital city $(n=34,35.4 \%)$ in the Municipality of Prishtina. A total of $62.6 \%$ of community pharmacies were located within the city, while $26.3 \%$ were near a healthcare center, and only $14.1 \%$ were outside the city. Half of the respondents $(n=50,50.5 \%)$ were between 23 34 age years old and $41.4 \%(n=41)$ between ages 35 and 49 years old. Concerning work experience, $46 \%(n=46)$ had less than ten years work experience while $42 \%(n=42)$ had between 10-19 years of work experience in community pharmacies and only 9\% with 30-39 years of experience and $3 \%$ more than 40 years of work experience. Given community pharmacy ownership, respondents revealed 
Jakupi A, Jakupi AB. Pharmacy practice architecture challenges in handling COVID-19 pandemic - sharing experience from a Kosovo pharmacy practice. Pharmacy Practice 2021 Oct-Dec;19(4):pagenumber.

https://doi.org/10.18549/PharmPract.2021.4.pagenumber

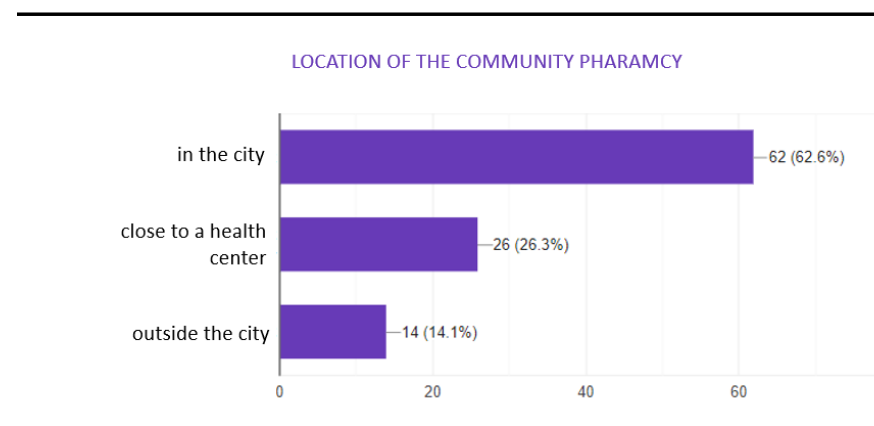

LOCATION OF THE COMMUNITY PHARAMCY

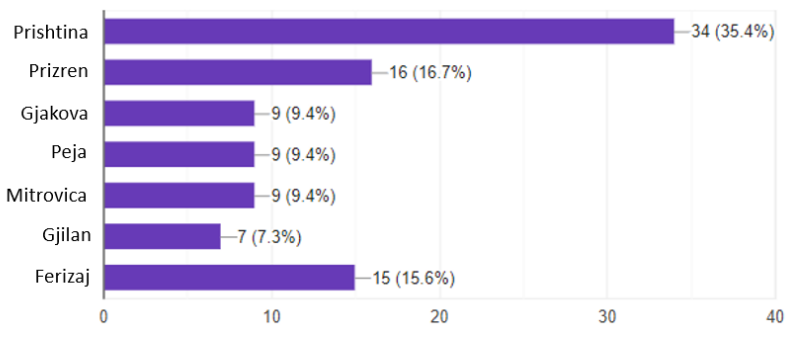

PHARMACISTS' DEMOGRAPHICS
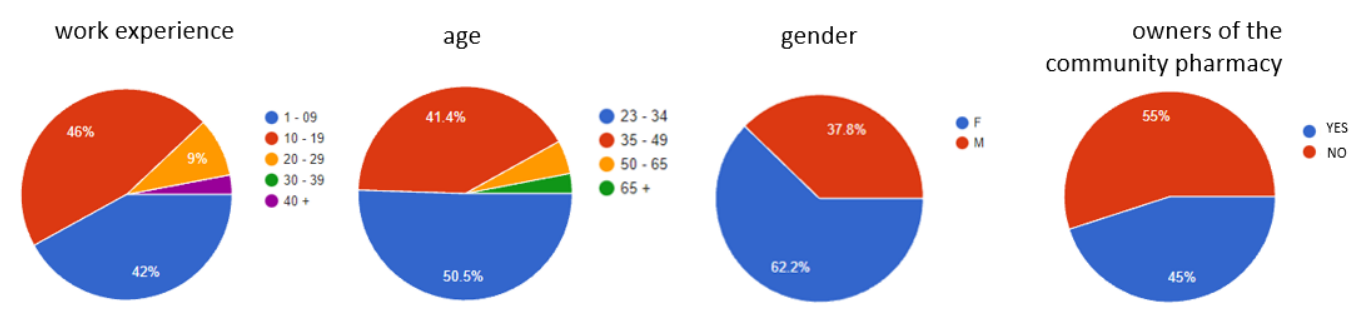

Figure 1. Pharmacists demographics with community pharmacy location

that more than half $(n=55,55 \%)$ were only hired as pharmacists while $45 \%$ owned their pharmacy.

The numbers of pharmacists presenting with different years of experience, with the breakdown of the pharmacist gender, together with community pharmacy location, are shown in Figure 1. (see. Fig. 1)

The first section of the medical management domain revealed service delivery to respond and serve the community and meet their medical needs, primarily by understanding the number of professionals and other employees that ensure efficient workflow. Respondent revealed that most community pharmacies ( $n=64,64 \%$ ) have only one pharmacist, with $27 \% \quad(n=27)$ having two pharmacists and $9 \%$ more than two pharmacists employed. As part of the professional personnel, community pharmacies have employed one pharmacist technician $(n=39,39 \%)$ or two $(n=33,33 \%)$. The main bearer of the community pharmacy workload falls upon the pharmacist. Therefore, to ensure the workflow of the service at the community pharmacies, it is important to understand the well-being of the pharmacist in the realm of an area where they can have a rest / a lunch break. This question discovered that $45.5 \% \quad(n=45)$ do their break at the administrative area as a shared area of an office and leisure. In comparison, 31.3\% $(n=31)$ do not have a specific area, and $21 \%(n=21)$ have a separate area dedicated to the activity, with $2 \%$ not having a lunch break. Following the further explanation, if there were any changes to these specific areas due to the pandemics, $87.9 \%(n=87)$ of the respondent had no change, and only $12.1 \%(n=12)$ had created new additions dedicated to the pharmacists. However, the future requests are oriented toward the creation of new spaces, dedicated: to leisure $(n=79,82.3 \%)$, kitchenette $(n=39,40.6 \%)$, meditation $(n=11,11.5 \%)$, fitness $(n=5,5.2 \%)$, office $(n=2,2 \%)$. Another aspect of the delivered service is related to the working hours, whereas $44 \%$ have changed the schedule

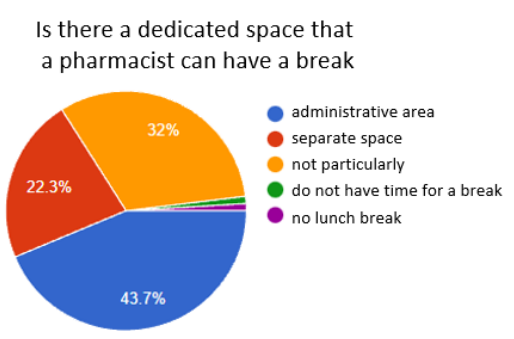

Was there additional space provided during COVID-19 peak for staff to take a break?

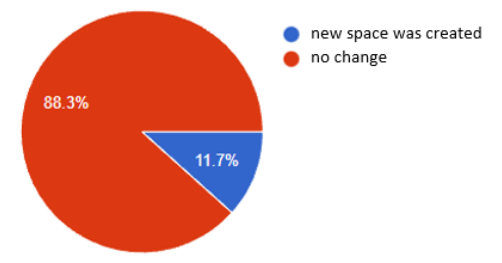

\begin{tabular}{|c|c|c|}
\hline \multicolumn{3}{|c|}{$\begin{array}{l}\text { Do you think the community pharmacy should } \\
\text { have space dedicated to the well-being of the } \\
\text { pharmacist? }\end{array}$} \\
\hline leisure & $n=81$ & $81.8 \%$ \\
\hline meditation & $n=11$ & $11.1 \%$ \\
\hline fitness & $n=5$ & $5.1 \%$ \\
\hline kitchenette & $n=40$ & $40.4 \%$ \\
\hline $\begin{array}{l}\text { no, there is } \\
\text { no need }\end{array}$ & $n=4$ & $4.1 \%$ \\
\hline office & $n=2$ & $2.1 \%$ \\
\hline
\end{tabular}

Figure 2. Dedicated area for the wellbeing of the pharmacist 
Jakupi A, Jakupi AB. Pharmacy practice architecture challenges in handling COVID-19 pandemic - sharing experience from a Kosovo pharmacy practice. Pharmacy Practice 2021 Oct-Dec;19(4):pagenumber.

https://doi.org/10.18549/PharmPract.2021.4.pagenumber

INFECTED PATIENTS

Is there separate area for consultation with patients?

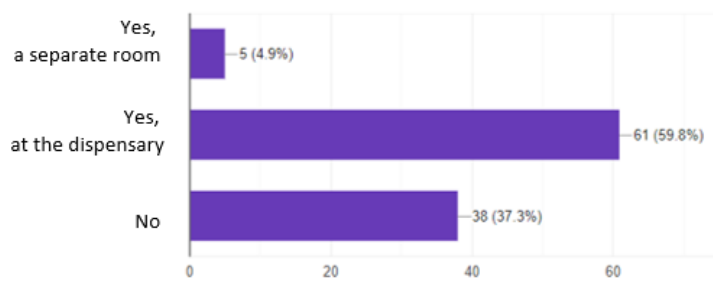

Figure 3. Treatment of the infected patients
Have you taken specific measures to dispense drugs to infected patients? What?

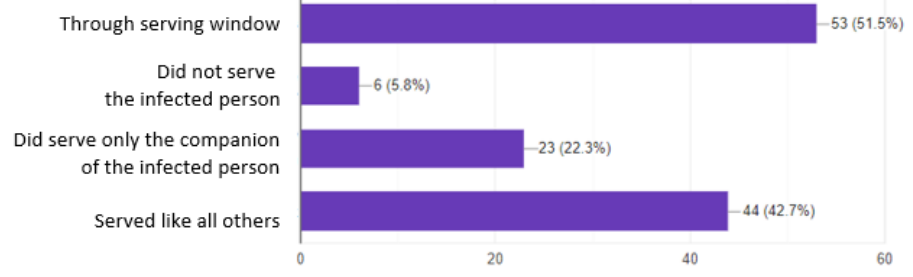

only during the peak of the pandemics, and $26 \%$ are continuing with the modified working hours. As an answer to this alternative, the respondents ( $n=61,66.3 \%)$ do not think that the prolonged working hours should remain as such, and $28.3 \%(n=26)$ favor the changed working schedule. Due to the risk of infection, some of the random pharmacy services were reduced, such as the measurement of the: blood pressure $(n=67,72 \%)$, glycemic ( $n=61,65.6 \%)$, cholesterol $(n=40,43 \%)$, weight and height $(n=37,39.8 \%)$ while $8 \%$ never offered this kind of service. However, community pharmacies added new services due to COVID 19, such as temperature measuring $(n=46,59.7 \%)$, rapid testing $(n=37,48.1 \%)$, and $16 \%$, which did not offer any additional services. And, as a response to the new additional services, some pharmacists ( $n=35,40.2 \%)$ favored adapting them in the long run, while the others $(n=47,54 \%)$ would go back to the pre-COVID routine. The main offered service to the patient remains the drug dispensing. The respondents revealed that nearly half of the community pharmacies ( $n=42,42.4 \%$ ) had served the infected patient the same as the others. In comparison, $51.5 \%(n=51)$ have delivered the drug through the small window, and $22 \%$ would serve only the companying person of the infected patient. The difficulties encountered for the implementation of COVID 19 measures were due to inventory arrangement $(n=34$, $37.8 \%)$, physical separation of the space $(n=23,25.6 \%)$, small area $(n=8,8.9 \%)$. However, community pharmacies $(n=28,28 \%)$ could accommodate the requested measures with no evident problem. During the pandemic time, the infected persons did visit the pharmacies, and it was discovered that most of the pharmacies ( $n=58,59.2 \%)$ did attain a separate consultation area for the patient at the end of the dispensary, while a separate consultation room for the patient could be found at $6 \%$ of the respondent, and with $26 \%$ of not having a consultation area at all. The provided seating for the patient could be easily used in advantage during the pandemic times, in which case the Kosovo community pharmacies showcased provision with inside seating $(n=73,73 \%)$ and outside seating $(n=6,6 \%)$, and on cases with no seating at all $(n=26,26 \%)$. At the same time, some pharmacies worldwide have tried e-pharmacy and home delivery as a solution to the pandemic precautions. Kosovo is not such a case due to legal restrictions. However, most pharmacists believe that digital technology in community pharmacies will change the medical management $(n=53,54.6 \%)$ and consultation manner ( $n=53,54.6 \%)$, with $26 \%$ attributing the architecture modification. In comparison, $20 \%$ do not believe that there will be any change. The pandemic exposed the need for additional multifunctional area $(n=78,79.6 \%)$, that could be used as a separate consultation room $(n=70,79.5 \%)$, telepharmacy $(n=31,35.2 \%)$, video presentations $(n=29$, $33 \%)$ or virtual communications $(n=30,34.1 \%)$, or other options $(n=15,17 \%)$.

Information domain - the second section is central to acknowledging pharmacists as information professionals especially, as the pandemic has highlighted pharmacists as a trusted information source providing valuable input for guidance, education, and policy. The precaution measures information on COVID 19 is received by $98 \%$ of the community pharmacies $(n=98,98 \%)$ from different national and international institutions. With Kosovo Pharmacist Chamber ( $n=87,87.9 \%)$ and Ministry of Health $(n=79,79.8 \%)$ being among the most important, together with National Institute of Public Health $(n=65$, $65.7 \%)$ and other international institutions ( $n=34,34.3 \%)$. On the other hand, the communication of the precaution measures to the patient was provided mostly with posters, markings ( $n=87,88.8 \%$ ) inside the store, by putting them in front store windows $(n=96,96 \%)$, floor $(n=60,60 \%)$, dispensary $(n=61,61 \%)$, walls $(n=11,11 \%)$. For patient information purposes, digital platforms are used as well: social media such as Facebook and Instagram $(n=32$, $32.7 \%)$, pharmacy websites $(n=16,16.3 \%)$, and phone messages via Viber and WhatsApp ( $n=11,11.2 \%)$.

The public health domain - shows the emerging pharmacists' roles in public health and is concerned with protecting and promoting safety, while the COVID-19 pandemic has enhanced this acknowledgment. First, by ensuring patient safety by keeping the social distance inside the pharmacy with two to three patients $(n=42$, $42 \%)$ at a time, or one at a time ( $n=37,37 \%)$. The reduced patient number allowed inside the pharmacy derives from 
Jakupi A , Jakupi AB. Pharmacy practice architecture challenges in handling COVID-19 pandemic - sharing experience from a Kosovo pharmacy practice. Pharmacy Practice 2021 Oct-Dec;19(4):pagenumber.

https://doi.org/10.18549/PharmPract.2021.4.pagenumber

many factors, including the separate entrance and exit provided within the pharmacy $(n=14,14.3 \%)$. Still, most community pharmacies use one opening as an entrance and exit ( $n=85,85.7 \%)$, with no separate circulation routes. Since the modern pharmacy design promotes a friendlier atmosphere with no glass separation in the dispensing area, it is COVID 19 that highlighted this particular division. In contrast, Kosovo pharmacists revealed that they always had the glass protection from when the pharmacy was opened $(n=71,71.7 \%)$, while $23.2 \%$ obtained glass protection with COVID outbreak. Concurrently with patient safety comes pharmacist's safety, whereas the survey discovered that most of the respondents were protected with mask and protection glass $(n=80,80 \%)$. With the provision of the anti-COVID vaccine, the study shows that $96 \%$ of the pharmacists were vaccinated $(89.9 \%$ with two doses and $7.1 \%$ only one dose), and $4 \%$ were not vaccinated. The most delicate and infection risk-prone activity was the exchange of the drugs and the money between the pharmacist and the patient. In which case, it was understood that the handling was done as usual with direct exchange $(n=60,62.5 \%)$ and in cases with separate basket or box $(n=32,33.3 \%)$. The most random needed patient anti COVID products, were provided free of charge, such as: alcohol ( $n=39,40.2 \%)$, masks ( $n=36,37.1 \%)$, temperature measurement $(n=54$, $55.7 \%)$ and water $(n=59,60.8 \%)$. Pharmacists' safety means physical and psychological safety from physical or verbal abuse from the patient. In these cases, the study tried to understand if pandemic has triggered these kinds of activities. Due to different reporting's around the world, the study exposed that Kosovo is no exception, with slightly half of the respondents stating that the abuse has been more evident during or due to COVID $(n=45,45.5 \%)$, and $53.5 \%$ did not notice any change of this matter. Since the focus of this research is about the built environment of the community pharmacy, questions about the space and available area discovered that most of the general area of the pharmacy is much higher $(n=70,70 \%)$ than the minimum $(n=30,30 \%)$ requested by the Kosovo laws. Same with the retail area $(n=59,60.2 \%)$ and administrative area $(n=78,79.6 \%)$, bigger than the requested dimensions. Due to the good possibility of the openings such as windows and doors, it was found out that the air ventilation and filtration, and humidity due to COVID 19 are treated with frequent natural ventilation $(n=84,84 \%)$ and air conditioning $(n=47,47 \%)$. Most expressed the UVGI (Ultraviolet germicidal irradiation) need in the community pharmacy for a virus-free space ( $n=66,66 \%)$.

Accordingly, to the view that the most considerable prone infectious space is the dispensary $(n=81,81.8 \%)$ and the entrance $(n=71,71.7 \%)$, respondents favored the automatic door as a solution with no touch opening $(n=62,63.3 \%)$. A lesson learned from COVID measures revealed that most pharmacists prefer an external provisional module that could be placed outside the pharmacy or adjacent to it $(n=39,40.2 \%)$ as an emergency response to similar future cases. When asked about the built environment interior preferences, it is revealed that the tendency to a more formalized, white design pharmacies is preferred rather than cozy/non formalized ( $n=31,32 \%$ ) ones.

\section{DISCUSSION}

This paper discusses the Kosovo pharmacy practice during the COVID-19 pandemic in regards to several safety precautions measures implemented through pharmacy design space modifications. We attempt to share the Kosovo experience of pharmacy practice. Some realizations and trials may be considered as a guidance and lesson for designing, adopting the appropriate space for emergency preparedness planning to many other countries to improve the public's provision of health care services. Our outcomes showed that COVID-19 had enforced a considerable burden on the Kosovo pharmacy system, and pharmacists are also experiencing this challenge in the pharmacy premises. ${ }^{20}$ This study aimed to explore the services provided by community pharmacies in Kosovo, a country with a high prevalence of COVID-19 cases (compared with other European countries). The sample size of pharmacies in this survey $(n=104)$ is satisfactory based on a recent study wherein it was reported that the total number of community pharmacies in Kosovo is 650 (KPC, 2021). The aim is to understand the new needs of the pharmacy premises.

In contrast, architects will need to create a new language in which to give these medical requirements substance and at the same time incorporate all the latest thinking about design for well-being. However, community pharmacies are rapidly adapting their frontline service to meet unprecedented demand as the pandemic develops. The research results confirmed the Kosovo pharmacist's data, same as compared with the data obtained in KPC, which highlight the profession of the pharmacist to be the newest among the medical jobs, bearing that the first pharmacy program is opened in 1996. Beforehand the pharmacy community was educated outside Kosovo in the region. The group age of the pharmacists consists $50.5 \%$ being between ages $23-34$ years old, while $41.4 \%$ being between ages 35-49 years old, which explains the easiness to the adaption, and willingness for new modern additions. The community pharmacy ownership revealed different approaches and perceptions toward the changes, while the respondent who owned the pharmacy $(45 \%)$ were more prone to comply with the requested changes.

The medical management domain explained the ability of pharmacists to ensure an efficient workflow of medical delivery and patient services during the COVID 19. Whereas only with a sufficient number of employees the community pharmacy could overcome the burden. Bearing that most pharmacies work from $8-20$, with $64 \%$ of them 
Jakupi A , Jakupi AB. Pharmacy practice architecture challenges in handling COVID-19 pandemic - sharing experience from a Kosovo pharmacy practice. Pharmacy Practice 2021 Oct-Dec;19(4):pagenumber.

https://doi.org/10.18549/PharmPract.2021.4.pagenumber

having only one pharmacist in charge for the 12-hour daily working shift and 39\% one pharmacy technicians. During the pandemics, the community pharmacies had more prolonged working hours. It explains the high pointed request of the pharmacist to have a separate room for leisure $(82.3 \%)$, kitchenette $(40.6 \%)$, or/and meditation (11.5\%). The much-needed rest or eventual break from a 12-hour schedule is done in the common administrative area of the pharmacy (45.5\%), while $31.3 \%$ do not have a specific place for leisure or rest. The workload with such extended working hours can affect the efficiency of the delivered services, consequently the medical management domain. The usual routine for some of the services has been changed due to pandemic emergency by reducing the measurement of the blood pressure (72\%), glycemic (65.6\%), cholesterol (43\%), weight and height (39.8\%), and at the same time adding new services, such as the temperature measurement $(59.7 \%)$ and rapid testing $(48.1 \%)$. Bearing that all of these services request an adequate space for an acceptable delivery, it comes down to understand that the newly offered services (as a response to COVID 19) require a more isolated space than the standard abovementioned reduced services. The latter, especially considering that $42 \%$ of the respondents favored permanently adapting the new additional services. Despite the clear guidelines about the COVID 19 precautions measures of the majority of the community pharmacists (98\%), an important fact revealed that $42.4 \%$ had served the infected patient the same as the others, with $51.5 \%$ have dispensed the drug through the serving window. The statistics show the architectural limitation of providing an alternative medicine dispensing besides the traditional one inside the store. The majority of the pharmacies are located within the city (62.6\%), which explains the space limitations of having a drive-through in the overbuilt central areas, as well as the restrictions of having a dispensing window, due to the already established floor plan organization (37.8\%), physical space division (25.6\%) and a small area (8.9\%). Only $28.28 \%$ of the community pharmacist could accommodate the requested COVID 19 measures without evident architectural obstacles. Despite the favoring factor of the community pharmacies, with having a more extensive area $(n=70,70 \%)$ than the one requested by the Kosovo Legislation, it exposes the lack of proper space organization. In medical health buildings, space flexibility is the key to a positive change and quick response to the new needs. Another vital piece of information demonstrated the need for a separate consultation area. With reference to $42.4 \%$ of community pharmacies serving the infected persons same as the others, with only $6 \%$ having a separate isolation room and $59.2 \%$ having a nominal separated area at the end of the dispensing area, it reveals an inadequate precaution for the infected persons. Respondents were aware of the need for an isolation room, with $79.5 \%$ of them requesting one as such. Community pharmacists understand the need for new pharmacy design and architecture approaches, such as modifying existing facilities to quickly create isolation and contamination zones.

The pharmacist is perceived as a trusted information source for guidance, education, and policy. The information domain has scored the highest performance service/activity, with $98 \%$ of the pharmacists being well informed, mainly from the national institutions. These sources of information are primarily used via the electronic format due to the pandemic; otherwise, other forms of communication like congresses and workshops are also common for this purpose. A study by Jakupi et al. 2019 revealed that pharmacists updated their knowledge mainly through the internet and relied on other methods, e.g., continuing education and discussions with colleagues. For patient information purposes were used mostly posters (96\%) and markings (88.8\%), exposed on many pharmacies built and inventory elements, such as front windows $(96 \%)$, dispensary (61\%), floor $(60 \%)$, walls $(11 \%)$. The willingness to reach the most remote patient with the needed information and education was also used digital platforms (32\%). Despite the desire of the young generation of the pharmacist to get good use of the digital technology advantages (80\%), the Kosovo Law does not allow home delivery through e-pharmacy, even though $54.6 \%$ believe that digital technology will change the drug distribution and consultation manner. Further, $35.2 \%$ of the respondents indicated the need for a multipurpose room that could be used, among others, for tele-pharmacy, virtual consultation, and video presentation.

The public health domain was achieved by implementing social distancing when trying to protect the patient, whereas $37 \%$ would allow one patient at a time, and $42 \%$ would allow two to three patients in a store. It could be explained by a lack of separate entrance and exit doors $(85.7 \%)$ in the pharmacy, which would regulate the adequate circulation and way finding of more than one patient inside the pharmacy. The most random anti COVID products were provided free of charge, such as alcohol $(n=39,40.2 \%)$, masks ( $n=36,37.1 \%)$, temperature measurement $(n=54,55.7 \%)$, and water $(n=59,60.8 \%)$, with all of them having no architectural obstacle of being implemented. Concurrently with patient's safety comes the pharmacist safety, in which case it was revealed that $80 \%$ of respondents were protected with a mask and the protection glass, whereas $71.7 \%$ stated that the protection glass in the dispensary was part of the interior and in pre-pandemic times. Nowadays, the COVID vaccination achieves safety, with $96 \%$ of the pharmacists being vaccinated. The most fragile areas that were prone to a high-risk infection were the dispensing process $(81.8 \%)$, where the exchange of medicine and money was done directly $(62.5 \%)$, and the entrance with the manual pushing/touching door (71.7\%). Both of these areas reveal the need for modification, whereas the 
Jakupi A , Jakupi AB. Pharmacy practice architecture challenges in handling COVID-19 pandemic - sharing experience from a Kosovo pharmacy practice. Pharmacy Practice 2021 Oct-Dec;19(4):pagenumber.

https://doi.org/10.18549/PharmPract.2021.4.pagenumber

first one could solve with card payment and basket medicine delivery, and the latter with automatic doors. Due to the excellent possibility of the openings such as windows and doors, it is revealed that the air ventilation, filtration, and humidity due to COVID 19 were treated with frequent natural ventilation $(n=84,84 \%)$ and air conditioning ( $n=47,47 \%)$. The majority expressed the usage of the UVGI (Ultraviolet germicidal irradiation) in the community pharmacy for a virus-free space $(n=66$, $66 \%$ ). The questionnaire revealed that pharmacists continue to meet and honor the needs of their patients but with the additional precautions and services adopted due to COVID 19 pandemic. ${ }^{7}$ As an answer to the rigidity of the internal organization, which is not adaptive to the new emergency needs, $40.2 \%$ declared the necessity to have an external provisional module as an emergency response to similar future cases.

\section{LIMITATIONS OF THE STUDY}

A higher response rate would be desirable to account for no respondents who potentially do not share the same views considering 800 pharmacies licensed from Kosovo Medicines Agency. Another potential limitation could be the applicability of the findings in the context of other countries where pharmacists practice according to different regulative specifications, although Covid is new for all. The focus of this study, however, was on issues that are new to pharmacists in Kosovo, just like they are new to pharmacists elsewhere, and the FIP universally recommends most of the measures reported here.

\section{CONCLUSION}

This article recaps how Kosovo pharmacists have implemented guidelines to the rapidly changing pandemic to guarantee patient and staff safety and the methods community pharmacists across Kosovo are taking in response to COVID-19. The goal was to compile knowledge, lessons learned and formulate concepts for long-term design solutions for new construction. The pharmacists will need to understand what are the unique needs. The flexibility of space will be the key to a positive change. An important lesson from the pandemic is that modern pharmacies often lack the flexibility to accommodate an emergency, and they should learn to create spaces that can be used alternatively. Consequently, the pharmacy profession needs to build upon the lessons and experiences of this global pandemic. Critically vital resources include equipment and staff, but built environments need to be considered equivalent. From a broader public policy standpoint, the design of our pharmacies should be done differently in the future to cope with the threat of future pandemics. It is a public health crisis rapidly becoming a health care/medical crisis, and the built environment should be an essential part of the solution. Therefore, it is necessary to understand the rituals of the local community pharmacists, how people move through space and use it, and use this knowledge to shape the areas and create future opportunities. Our present-day situation marks a moment that will surely change the way we consider designing and building the spaces we need in the future. Architecture has been referred to as a passive solution. Still, if it is positioned as an active mediator in the fight against COVID 19, it can survive this emergency and the next one. Although the pandemic exposed many errors in the Kosovo community pharmacy system, it has also discovered a unique possibility for collaboration and novelty across the professions, working on redesigning the future community pharmacy environment, first and foremost on guaranteeing patient and staff safety. If community pharmacy is designed purposefully, it can contribute to the prevention and control of future pandemic situations, including COVID-19.

\section{DECLARATIONS}

\section{ETHICS APPROVAL AND CONSENT TO PARTICIPATE}

The Kosovo Pharmaceutical Chamber committee approved the study

\section{CONSENT FOR PUBLICATION}

Not applicable

\section{AVAILABILITY OF DATA AND MATERIALS}

All data generated or analyzed during this study are included in this published article

\section{FUNDING}

This research did not receive grants from any funding agency in the public, commercial, or not-for-profit sectors.

\section{COMPETING INTERESTS}

The authors declare that they have no known competing financial interests or personal relationships that could have influenced the work reported in this paper.

\section{AUTHORS' CONTRIBUTION}

This article is interdisciplinary. Therefore each of the authors was focused on part dealing with their specialized area. Dr. Arta Basha-Jakupi's contribution is related to the architecture realm, while Dr. Arianit Jakupi has community pharmacy regulations and legislation. Thus, this research is a product of joint involvement.

\section{ACKNOWLEDGMENTS}

The authors would like to acknowledge all pharmacists in Kosovo for participating in the study. 
Jakupi A, Jakupi AB. Pharmacy practice architecture challenges in handling COVID-19 pandemic - sharing experience from a Kosovo pharmacy practice. Pharmacy Practice 2021 Oct-Dec;19(4):pagenumber.

https://doi.org/10.18549/PharmPract.2021.4.pagenumber

\section{References}

1. World Health Organization. WHO announces COVID-19 outbreak a pandemic. 2020

2. Adam J.P, Khazaka M, Charikhi F, et al. Management of human resources of a pharmacy department during the COVID-19 pandemic: Take-aways from the first wave. Research in Social and Administrative Pharmacy. 2021;17(1):1990-1996. https:// doi.org/10.1016/j.sapharm.2020.10.014

3. Centers for Disease Control and Prevention. Guidance for pharmacies: guidance for pharmacists and pharmacy technicians in community pharmacies during the COVID-19 response. 2020

4. World Health Organization. The legal and regulatory framework for community pharmacies in the WHO European Region. 2019

5. Watson K.E, Schindel T.J, Barsoum M.E, et al. COVID the Catalyst for Evolving Professional Role Identity? A Scoping Review of Global Pharmacists' Roles and Services as a Response to the COVID-19 Pandemic. Pharmacy 2021;9(2):99. https://doi. org/10.3390/pharmacy9020099

6. Pinto G.S, Hung M, Okoya F, et al. FIP's response to the COVID-19 pandemic: Global pharmacy rises to the challenge. Research in Social and Administrative Pharmacy. 2021;17(1):1929-1933. https://doi.org/10.1016/j.sapharm.2020.07.004

7. Stewart K, Barai R, Priarities N. How to keep your community pharmacy running during the COVID-19 pandemic. Pharm J. 2020. https://doi.org/10.1211/pj.2020.20207855

8. Street G. The Pharmacy Guild of Australia. 2020

9. Hercules W.J, Anderson D.C, Sansom, M. Architecture-ACritical Ingredient of Pandemic Medicine: An Open Letter to Policy Makers. HERD: Health Environments Research \& Design Journal. 2020;13(3):247-252. https://doi.org/10.1177/1937586720928432

10. Budds D. Design in the age of pandemics. Curbed. 2020

11. Bahlol M, Dewey R.S. Pandemic preparedness of community pharmacies for COVID-19. Research in Social and Administrative Pharmacy. 2021;17(1):1888-1896. https://doi.org/10.1016/j.sapharm.2020.05.009

12. Eikenberry S.E, Mancuso M, Iboi E, et al. To mask or not to mask: Modeling the potential for face mask use by the general public to curtail the COVID-19 pandemic. Infectious Disease Modelling. 2020;5:293-308. https://doi.org/10.1101/2020.04.06.20055624

13. Zhu N, Zhang D, Wang W, et al. A novel coronavirus from patients with pneumonia in China, 2019. New England journal of medicine. 2020

14. Waite R, Pitcher G. How will Covid-19 change the design of health facilities? Architects' Journal. 2021

15. Ministry of Health. Administrative Instruction (Health). Kosovo 2015

16. Hoti K, Jakupi A, Hetemi D, et al. Provision of community pharmacy services during COVID-19 pandemic: a cross sectional study of community pharmacists' experiences with preventative measures and sources of information. International journal of clinical pharmacy. 2020;42(4):1197-1206. https://doi.org/10.1007/s11096-020-01078-1

17. Kosovo Statistics Agency. Kosovo Census 2011

18. Ministry of Health. Numri i të vaksinuarve në Kosovë. 2021

19. Ministry of Health. Aktivitetet në lidhje me monitorimin, parandalimin e rrezikut të përhapjes së COVID-19. 2020

20. Parajuli D.R, Khanal S, Wechkunanukul K.H, et al. Pharmacy practice in emergency response during the COVID-19 pandemic: Lessons from Australia. Research in Social and Administrative Pharmacy. 2021. https://doi.org/10.1016/j.sapharm.2021.08.013

21. Albutt K, Luckhurst C.M, Alba, et al. Design and impact of a COVID-19 multidisciplinary bundled procedure team. Annals of surgery. 2020;272(2):e72. https://doi.org/10.1097/sla.0000000000004089

22. Jakupi A, Raka D, Kaae s et al. Culture of antibiotic use in Kosovo-an interview study with patients and health professionals. Pharmacy Practice. 2019;17(3). https://doi.org/10.18549/pharmpract.2019.3.1540

23. World Health Organization. WHO health emergency dashboard. WHO (COVID-19) homepage. 2020 